\title{
Special issue on: constraint programming approaches to combinatorial optimization
}

\author{
Louis-Martin Rousseau Willem-Jan van Hoeve
}

Published online: 23 July 2014

(C) EURO - The Association of European Operational Research Societies 2014

This special issue of the European Journal of Computational Optimization focuses on papers that contribute to the field of constraint programming (CP) and its boundary with classical mathematical programming methods such as mixed integer programming (MIP).

This issue starts with a "Constraint Programming Primer", which presents an overview of the main CP techniques and key ideas used in the following papers.

The first paper following this primer, "Improving strong branching by domain propagation", investigates how $\mathrm{CP}$ techniques such as propagation can be useful to speed up a classical MIP procedure such as strong branching. The paper demonstrates the usefulness of this combined approach on instances of the MIP problem library.

The following three papers propose novel methodologies that are applied in the context of real industrial applications. "Bound-Consistent Spread Constraint, Application to load balancing in nurse to patient assignments" proposes a global constraint that allows restricting the distribution of values occurring in a vector of variable to spread them as evenly as possible. A bound consistency technique is introduced and an application to the field of nurse rostering is proposed.

The fourth paper "CP Methods for Scheduling and Routing with Time-Dependent Task Costs" proposes a Lazy-Clause Generation CP model that is compared to direct $\mathrm{CP}$ and MIP models in the context of scheduling vessels in ports subject to strict tide constraints.

\section{L.-M. Rousseau ( $\varangle)$}

Mathématiques et Génie Industriel, C.P. 6079, succ. Centre-ville, Montréal, QC H3C 3A7, Canada e-mail: Louis-Martin.Rousseau@polymtl.ca 
Finally, "Solving Wind Farm Layout Optimization with Mixed Integer Programs and Constraint Programs" uses a hybrid MIP and CP method to capture the nonlinearity which is induced by the aerodynamic interactions among multiple wind turbines.

Together, these papers present a broad perspective of state-of-the-art CP methodologies that can be applied, also in combination with MIP techniques, to solve challenging real-world applications of combinatorial optimization problems. 